\title{
Camouflage Effect Evaluation of Pattern Painting Based on Moving Object Detection
}

\author{
Xianhui Rong, ${ }^{1,2}$, Qi Jia ${ }^{2, *}$, Weidong $\mathrm{Xu}^{2}$, Xuliang $\mathrm{Lv}^{2}$ and Jianghua $\mathrm{Hu}^{2}$ \\ ${ }^{1}$ Business School of Hohai University, Nanjing, China \\ ${ }^{2}$ College of Field Engineering, PLA University of Science and Technology, Nanjing, China \\ ${ }^{*}$ Corresponding author
}

\begin{abstract}
There is lack of effective method of camouflage effect evaluation of pattern painting of moving object. To evaluate the camouflage effect objectively and quantitatively, the method camouflage effect evaluation of pattern painting based on moving object detection is proposed. Three moving object detection methods of optical flow method, frame differential method and background subtraction method are compared, and the background subtraction method of updating background is used in the paper. The basic principle of dynamic camouflage effect simulation evaluation of pattern painting is proposed, and the software is implemented and compiled. The results show that the model is operability and accurate and it can be used in objectively camouflage effect evaluation in camouflage pattern painting of moving targets.
\end{abstract}

Keywords-moving target; target detection; camouflage pattern; camouflage effec; background subtraction

\section{INTRODUCTION}

The main factor affecting the camouflage effect is the level of coordination of target and background spot in surface spot shape, size, color and spatial distribution, and its quantitative evaluation index is the probability of finding the disguised object. Generally, detection probability need organizes a large number of observers' field observations under field conditions, and obtain after statistical analysis. The drawback is that funding is high and test repeatability is poor, which greatly limits the quantitative evaluation of target camouflage effect. Target camouflage effect evaluation includes both static and dynamic target state. All along, the research in this area focused on static and dynamic research for less. For a single military objective in terms of movement, dynamic camouflage effect is even more important than the static camouflage effect. Therefore, the objective evaluation of dynamic camouflage effect cannot be ignored.

\section{Moving TARget Detection Methods}

Moving object detection is an important research direction of computer vision [1, 2]. Moving object detection [3-5] is characterized by sequence analysis to detect the presence or absence relative moving target motion in video with background. At present, there are three main commonly used detection methods: the optical flow method [6, 7], the frame differential method [8] and the background subtraction method $[9,10]$.

\section{A. Optical Flow Method}

When the camera and the target have a relative motion, the movement of the optical characteristics of the target projects onto the image plane and form a optical flow. When the target is in motion, the target number, speed, distance, and shape information can be analyzed by optical flow method. By calculating the displacement vector optical flow tracking algorithm initialization, effectively extract moving target features that enable the detection of moving targets.

Here, set pixels values $g(x, y, t)$ in the image pixel point $(x, y)$ in time $t$, which is a dynamic function of time and space, according to the Taylor formula, we have

$$
g(x+d x, y+d y, t+d t)=g(x, y, t)+g_{x} d x+g_{y} d y+g_{t} d t+o
$$

where, $g_{x}, g_{y}, g_{t}$ is the number of partial derivatives direction $x, y, t$ respectively. The image has the appropriate adjacent points, which is

$$
g(x+d x, y+d y, t+d t)=g(x, y, t)
$$

Meanwhile, $d x, d y, d t$ are very small, there is

$$
g_{t}=-g_{x} \frac{d x}{d t} d x-g_{y} \frac{d y}{d t}
$$

Here, set the speed $c=\left(\frac{d x}{d t}, \frac{d y}{d t}\right)=(\mu, v)$. If the motion picture changing speed is slow, minimize the squared error variance can be simplified as

$$
E^{2}(x, y)=\left(g_{x} \mu+g_{y} v+g_{t}\right)^{2}+\lambda\left(\mu_{x}^{2}+\mu_{y}^{2}+v_{x}^{2}+v_{y}^{2}\right)
$$

where, $\lambda$ on behalf of Lagrange multiplier, $\mu_{x}^{2}, \mu_{y}^{2}, v_{x}^{2}, v_{y}^{2}$ denote the partial derivative of the error square. Thus, the definition of an average of speed $\bar{\mu}, \bar{v}$ on the field on $x, y$. 


$$
\left\{\begin{array}{l}
\left(\lambda^{2}+g_{x}^{2}\right) \mu+g_{x} g_{y} v=\lambda^{2} \bar{\mu}-g_{x} g_{t} \\
\left(\lambda^{2}+g_{y}^{2}\right) v+g_{x} g_{y} \mu=\lambda^{2} \bar{v}-g_{y} g_{t}
\end{array}\right.
$$

Solutions of this equation is that

$$
\left\{\begin{array}{l}
\mu=\bar{\mu}-g_{x} P / D \\
v=\bar{v}-g_{y} P / D
\end{array}\right.
$$

In the formula, $P=g_{x} \bar{\mu}+g_{y} \bar{\nu}, D=\lambda^{2}+g_{x}^{2}+g_{y}^{2}$.

\section{B. Frame Differential Method}

Frame differential method, also the time difference method, the difference is the algorithm to extract moving objects between images using image sequences adjacent. The method that the sequence of images in two adjacent regions have the same or similar lot, but those are not the same or similar area is considered a change in the region, which is the region where the moving object.

Set the size of the image sequence $m \times n$ in the frame $t$ image $G(t)$ represented by the pixel value $g(i, j, t)$ at the point $(i, j)$, then the frame $t$ difference resulting image of the frame $t-1$ image is

$$
D(t)=G(t)-G(t-1)
$$

Here, set image sequence grayscale, there is

$$
D(t)=\left[\begin{array}{ccc}
g(1,1, t)-g(1,1, t-1) & \cdots & g(1, n, t)-g(1, n, t-1) \\
\vdots & & \vdots \\
g(m, 1, t)-g(m, 1, t-1) & \cdots & g(m, n, t)-g(m, n, t-1)
\end{array}\right]
$$

To make the image difference after the difference more significant, the image makes be the value of 0 or 1 , then

$$
d(i, j, t)= \begin{cases}1 & |g(i, j, t)-g(i, j, t-1)| \geq T \\ 0 & |g(i, j, t)-g(i, j, t-1)|<T\end{cases}
$$

where, $T$ is the binary threshold value processing. Size threshold directly affect the outcome of moving target detection, select the appropriate threshold for inter-frame difference is crucial.

\section{Background Subtraction Method}

Background subtraction method, also known as background difference method, is a method of moving objects detected by the difference image sequence and the reference background model. Background subtraction need to enter the initial case scenario, that is the background of the scene, and then compare the observed scene and background scene, the background subtraction from the observed scene, get moving target. This method requires that the camera must remain static.
Set observed pixel value $g(i, j, t)$ is set at a point $(i, j)$ in time $t$, the pixel value $b(i, j, t)$ at the point $(i, j)$ of time $t$ represented by the background, then

$$
d(i, j, t)= \begin{cases}1 & |g(i, j, t)-b(i, j, t)| \geq T \\ 0 & |g(i, j, t)-b(i, j, t)|<T\end{cases}
$$

where, $T$ is the binary threshold value processing. In general, the background will happen with the site lighting and other minor changes, in order to weaken the possible changes in the background on target detection, background updates are timely and effective guarantee the reliability of the algorithm, the general approach to be mean filter solve.

\section{Comparison of Three Methods}

Currently, optical flow method, frame differential and background subtraction method are widely used to detect these goals. And these strengths and weaknesses are more obvious [11-13], which as shown in Table I below.

Currently, researchers have for the shortcomings of the three target detection algorithms are proposed solutions. Researchers tend to fusion of the three algorithms, whichever strengths, abandoned its shortcomings, to solve some problems, such as calculating the amount of anti-noise and background filtering, effectively improve the performance of moving target detection algorithm. However, in general, these algorithms are improved to decrease the cost of certain advantages, limitations still unavoidable. In order to accurately and quickly extract the moving target, using paper background updating achieves background subtraction method.

TABLE I. ADVANTAGES AND DISADVANTAGES OF THREE KINDS OF MOVING TARGET DETECTION METHODS

\begin{tabular}{|c|c|c|}
\hline Methods & Advantages & Disadvantages \\
\hline $\begin{array}{c}\text { Optical } \\
\text { flow }\end{array}$ & $\begin{array}{c}\text { The camera can be in } \\
\text { movement. Access to } \\
\text { information is rich. }\end{array}$ & $\begin{array}{c}\text { Large amount of } \\
\text { computation. Anti-noise } \\
\text { performance is poor. }\end{array}$ \\
\hline $\begin{array}{c}\text { Frame } \\
\text { differential }\end{array}$ & $\begin{array}{c}\text { A small amount of } \\
\text { Environmental } \\
\text { adaptability. }\end{array}$ & $\begin{array}{c}\text { Accuracy is poor. The } \\
\text { camera must be stationary. } \\
\text { Prone to the phenomenon } \\
\text { of empty. }\end{array}$ \\
\hline $\begin{array}{c}\text { Background } \\
\text { subtraction }\end{array}$ & $\begin{array}{c}\text { A small amount of } \\
\text { computation. Target } \\
\text { detection accurate. }\end{array}$ & $\begin{array}{c}\text { Sensitive to light and } \\
\text { changes in external } \\
\text { conditions. The camera } \\
\text { must be stationary. }\end{array}$ \\
\hline
\end{tabular}

\section{The BASIC Principles of DyNAMic SimUlation CAMOUFlage EFFECT EVALUATION}

Here, the dynamic camouflage effect evaluation uses background subtraction method. In simulation evaluation, the collected image of the scene is set as a background image. Camouflage pattern on the background image maps, and noise obtained after the processing target image. Background image and the target image are shown in Figure I. Figure I (a) shows the background image, (b) and (c) shows the two images comprise a moving object. Figure II shows the movement of the target area for the target detection obtained. 


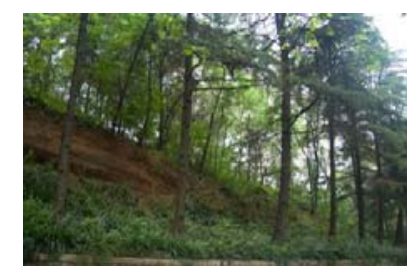

(a)

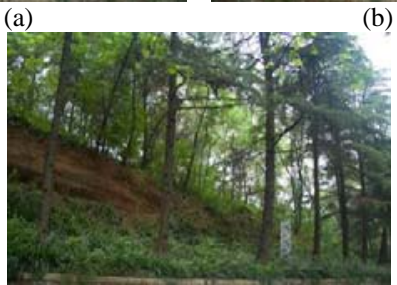

(c)

FIGURE I. BACKGROUND IMAGE AND THE TARGET IMAGES

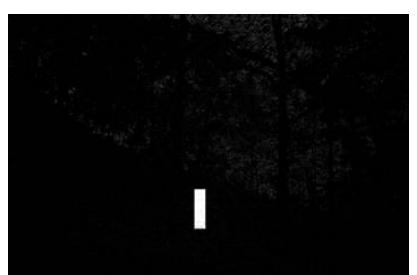

(a)

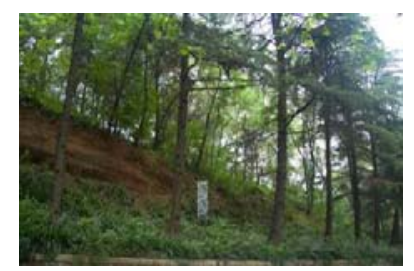

(b)

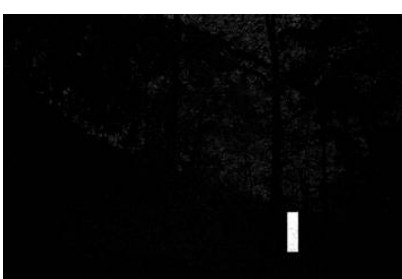

(b)
FIGURE II. MOVING TARGET AREA OBTAINED BY TARGET DETECTION

\section{Software Programming OF Dynamic CAMouflage EFFECT EVALUATION}

To facilitate the assessment of military targets analogy dynamic camouflage effect, the preparation of the dynamic simulation camouflage effect evaluation program, the user interface is shown in Figure III.

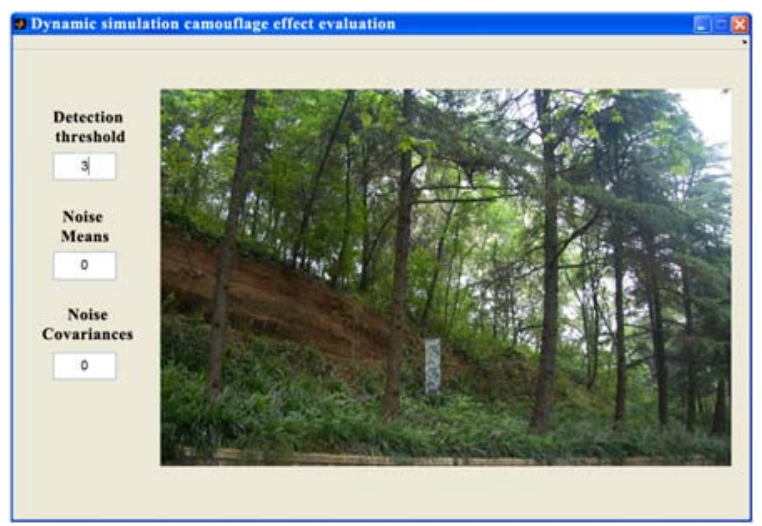

FIGURE III. USER INTERFACE OF DYNAMIC SIMULATION CAMOUFLAGE EFFECT EVALUATION

User interface of dynamic simulation camouflage effect evaluation software include the preparation of three large pieces: file action bar, the left area and the intermediate area. File action bar background and the main achievement of the target image read, save moving images and other control operations; the left area of the parameter reading area, including motion detection threshold value, the added
Gaussian noise of mean and variance; intermediate region of the display area, accounting for the largest proportion, realtime display of the image reading, and motion detection to obtain a moving image.

\section{EXPERIMENT AND DISCUSSION}

The use of the preparation of dynamic simulation camouflage effect evaluation software for simulation and evaluation of static camouflage effect of the selection of the best three programs organized dynamic effects simulation evaluation. Digital camouflage effects evaluation of dynamic simulation are shown in Figure IV, where in the Gaussian noise mean is set to 1 , the variance is 1 .

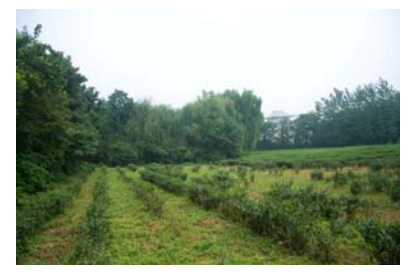

(a)

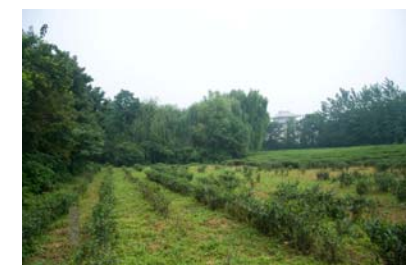

(b)

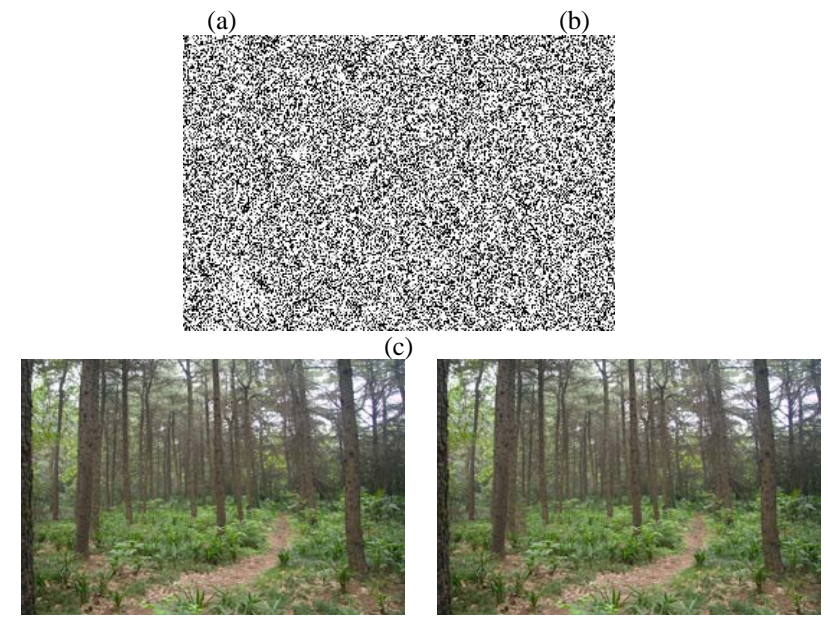

(e)

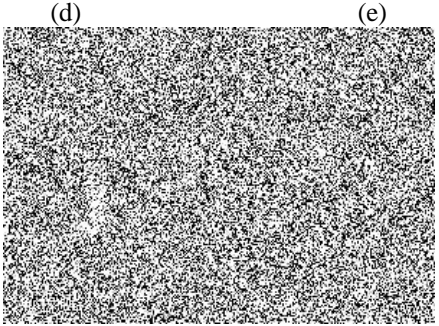

(f)

FIGURE IV. DYNAMIC SIMULATION CAMOUFLAGE EFFECT EVALUATION OF DIGITAL CAMOUFLAGE

It shows that in Figure IV in two different contexts, digital camouflage design scheme can achieve better dynamic camouflage effect. Simulation results of the evaluation show that under certain add noise conditions, static selection out of the three programs can effectively achieve optimum integration with the background, hidden in background noise, difficult to detect enemy reconnaissance. 


\section{CONCLUSION}

Camouflage pattern camouflage effect evaluation on a moving target, is an important aspect of the camouflage effect evaluation. To achieve the objective and quantitative evaluation of target camouflage effect, this paper based on moving object detection camouflage pattern camouflage effect evaluation. This paper compares the optical flow detection methods, features three kinds of moving target frame difference and background subtraction after. we decided to implement moving target detection using background subtraction method to update the background. This paper presents a dynamic simulation evaluation of the basic principles of camouflage effect, and the preparation of computer software implementation. Practical application results show that: the model has strong operability quantify accurate, and objective evaluation can camouflage pattern camouflage effect of moving targets. This article provides a moving target camouflage effect thinking, not only can be used to evaluate the effect of optical camouflage, camouflage can also evaluate the effect of infrared and radar provides a reference and reference.

\section{REFERENCES}

[1] K. X Dai, G. H. Li, D. Tu, J. Yuan, "Prospects and current studies on background subtraction techniques for moving objects detection from surveillance video,” Journal of Image and Graphics, vol. 11, pp. 919-927, 2006.

[2] M. Piccardi, "Background subtraction techniques: a review," IEEE International Conference on Systems, Man and Cybernetics, pp. 30993104, 2004.

[3] Z. Xu, P. Shi, “An eigenbackground subtraction method using recursive error compensation,” Advances in Multimedia Information ProcessingPCM 2006, pp. 779-787, 2006.

[4] Z. Xu, Y. H. Gu, P. Shi, "Recursive error-compensated dynamic eigenbackground learning and adaptive background subtraction in video,” Optical Engineering, vol.47, pp. 11-20, 2008.

[5] N. M. Oliver, B. Rosario, A. P. Pentland, "A bayesian computer vision system for modeling human interactions,” IEEE Transactions on Pattern Analysis and Machine Intelligence, vol. 22, pp. 831-843, 2000.

[6] S. Roth, M. J. Black, "On the spatial statistics of optical flow," Computer Vision 2005 IEEE International Conference ICCV2005, pp. 42-49, 2005.

[7] P. N. Pathirana, A. E. K. Lim, J. Carminati, "Simultaneous estimation of optical flow and object state, a modified approach to optical flow calculation,” Networking, Sensing and Control, 2007 IEEE International Conference, pp. 634-638, 2007.

[8] M. Durus, A. Ercil, "Robust vehicle detection algorithm,” IEEE Signal Processing and Communications Applications, pp. l-4, 2007.

[9] S. McKenna, "Tacking groups of People," Computer vision and Image Understanding, vol.80, pp. 42-56, 2000.

[10] C. Stauffer, W. Grimson, “Adaptive background mixture models for real-time tracking,” Proc IEEE Conference on Computer Vision and Patten Recognition, Fort Collins, Colorado, pp. 246-252, 1999.

[11] B. Li, "Study on Moving Object Detection and Tracking in Video Sequences,” Beijing: Beijing Jiaotong University, 2010.

[12] Y. Li, "Research on the algorithms of moving objects detection and tracking in intelligent monitoring system," Harbin: Harbin Engineering University, 2010.

[13] Z. B. Zhang, "Moving objects detection based on improved Gaussian mixture model,” Harbin: Harbin Engineering University, 2010. 